\title{
Comportamiento de Laminaciones Coplanares y No Coplanares en Acero API5L X52 Simuladas mediante Elementos Finitos
}

\author{
Alberto Servin ${ }^{1}$, Jorge L. González ${ }^{2}$ y Alfredo Morales ${ }^{3^{*}}$ \\ (1) Tecnológico de Veracruz, Metal-Mecánica, M.A. de Quevedo 2779, 91860 Veracruz-México \\ (2) Instituto Politécnico Nacional, ESIQIE, Av. Adolfo López Mateos, 07773 México D.F.-México \\ (3) Tecnológico de Puebla, Tecnológico 420, 72420 Puebla-México \\ (e-mail:alfredo.morales@mexssub.com)
}

*autor a quien debe ser dirigida la correspondencia

\begin{abstract}
Resumen
Se determina el comportamiento mecánico de laminaciones simples y escalonadas modeladas por elementos finitos en condiciones no lineales de esfuerzo-deformación, para comprender la secuencia de la interacción de los campos de esfuerzos en los extremos de la grietas. Se estableció una matriz de simulación variando la extensión circunferencial de las laminaciones, con espesor del tubo de $610 \mathrm{~mm}$ de diámetro, con separación vertical y horizontal para el caso de laminaciones escalonadas. Se determino la función de distribución de esfuerzos en la región de las laminaciones en función de la presión en la grieta y en el tubo. Se estableció la presión que origina alcanzar la cedencia y resistencia ultima del material API5L X52, determinando que las laminaciones simples cuya extensión circunferencial va de 24 a $127 \mathrm{~mm}$, soportan 50\% más de presión de cedencia que las laminaciones escalonadas, y el 100\% más de presión máxima permisible. Basados en la graficas presión versus radio de las laminaciones se concluye que las laminaciones no coplanares representan mayor riesgo de agrietamiento en una tubería.
\end{abstract}

Palabras clave: agrietamiento en tuberías, grietas escalonadas, elementos finitos, fractura, tuberías

\section{Behavior of Coplanar and Non-Coplanar Laminations in API5L X52 Steel Simulated by Finite Elements}

\begin{abstract}
The mechanical behavior of the coplanar and non coplanar laminations by finite element using non lineal material conditions was determined, with the purpose of knowing the interaction sequence of the stress fields in the crack tips. The analysis includes a matrix simulation considering the circumferential length lamination, wall thickness of the pipe $610 \mathrm{~mm}$ diameter, with vertical and horizontal distance for non coplanar laminations. The stress distribution in the laminations regions as a function of the internal pressures in the cracks and pipe were determined. The simulations done allowed defining the yield and the strength pressure that reach the mechanical properties of the API $5 \mathrm{~L}$ X52. It was found that coplanar laminations with 24 at $127 \mathrm{~mm}$ of circumferential length resist $50 \%$ more yield pressure than the non coplanar laminations and $100 \%$ more pressure than the maximum allowable. Based in the plots of pressure -vs- lamination radius it is concluded that non coplanar laminations have higher cracking risk in the pipeline.
\end{abstract}

Keywords: pipeline cracking, coplanar laminations, finite elements, fracture, pipelines 


\section{INTRODUCCIÓN}

Desde que Makio (1978), reporto haber analizado el comportamiento de grietas no coplanares mediante simulación por elemento finito. Estos análisis se han extendido al estudio del agrietamiento inducido por hidrógeno absorbido en tuberías de transporte de gas amargo (AlH), el cual se caracteriza por la formación de grietas totalmente contenidas en la pared de las tuberías y que se encuentran presurizadas por hidrógeno llamadas laminaciones. Ha sido demostrado por Zacaria y Davies (1993), que las laminaciones crecen y se interconectan por la presión del hidrógeno en su interior y que la interconexión se acompaña por una considerable deformación plástica y la fractura fuera del plano, situación influenciada por la presencia del esfuerzo externo.

Moussa (1991), analizan mediante MEF dos grietas no coplanares traslapadas y separadas en la vertical, sujetas a una carga de tensión, concluyendo la influencia que tiene la interacción del tamaño de grietas y esta separación, obteniendo una función cuadrática del tamaño de grieta. Noda (2001), calculó el factor de intensidad de esfuerzos para dos grietas semielipticas mediante el método de las fuerzas de cuerpo y reporto que la interacción es despreciable al estar traslapadas y que es altamente influenciada cuando una de las grietas es de mayor dimensión con respecto a la otra. Itou (2001), analizo en tres dimensiones un sistema de grietas cuadradas y paralelas bajo el impacto de un carga dinámica, el planteamiento matemático lo realiza en función de Laplace y Fourier y resuelve mediante ecuaciones integrales, determinando que mientras más próximas se encuentren las grietas en la dirección vertical mayor es el factor de intensidad. Kratzig (2001), investigó el nivel del daño estructural en un punto de un sistema mecánico aplicando la mecánica de sólidos e introduce un indicador de daño con un sistema no lineal de ecuaciones. La plastificación y agrietamiento del extremo de una laminación simple o del espacio interlaminar es un paso previo a la falla, el análisis propuesto puede establecer las bases para un criterio de evaluación. Gonzalez (2004), determino que el mecanismo de falla en tubos con laminaciones al incrementar la presión interna, se lleva a cabo por la interacción de los campos de esfuerzo en la zona interlaminar así como por la plastificación del ligamento, como se muestra esquemáticamente en la figura 1. En esta figura se incluye una fotografía de un tramo de tubo con AlH que fue presurizado hasta la falla, donde se observa la fractura escalonada resultante de este mecanismo.

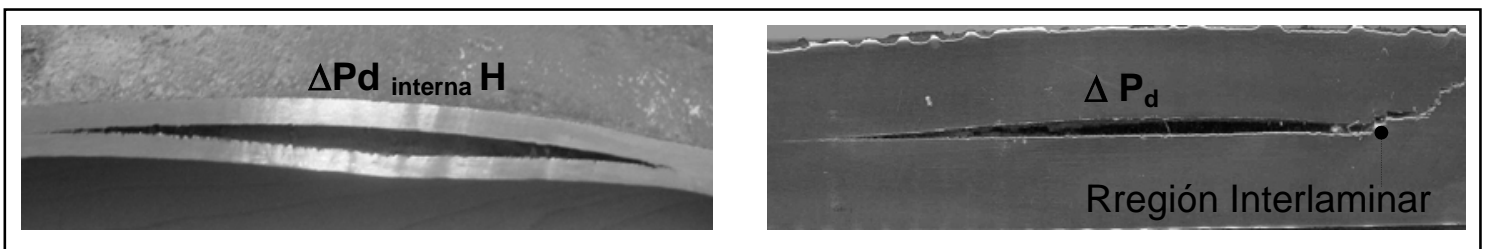

Fig. 1: Fotografía de dos ductos respectivamente con laminación simple y escalonada.

Morales (2008), establece siguiente secuencia: una primera etapa, la presión en el interior de la grieta hace que se alcance la cedencia del material y posteriormente la resistencia máxima, lo que se manifiesta en la extensión circunferencial de la laminación simple, como se muestra en la figura 2 (a). Para el caso de laminaciones escalonadas, adicionalmente habrá una interacción de los campos de esfuerzos de las puntas de grieta convergentes, como se muestra en la figura 2 (b).

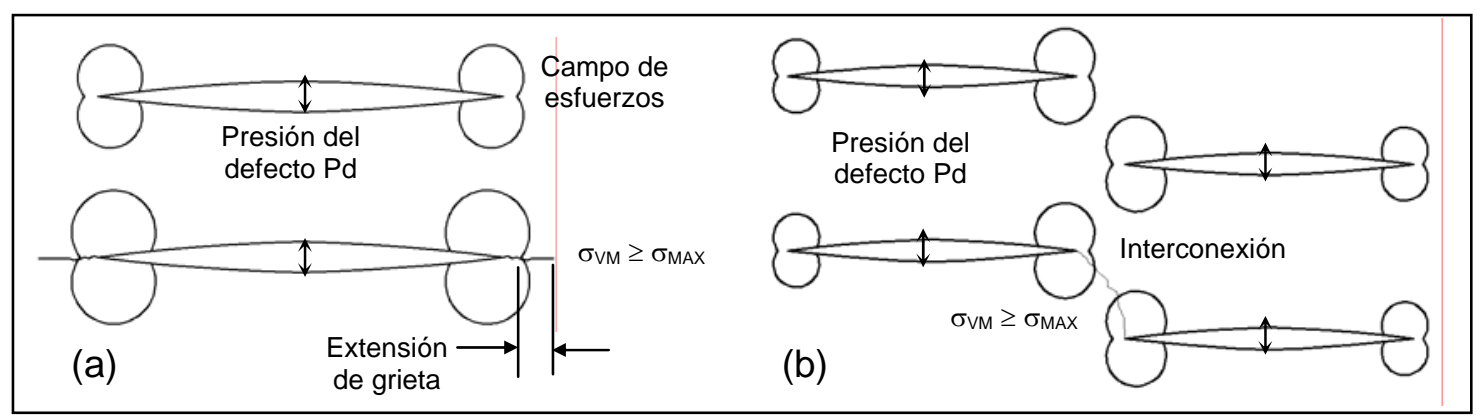

Fig. 2: Representación esquemática de la extensión de una laminación simple (a) y la interconexión de una laminación escalonada por el mecanismo de presión (b). 


\section{METODOLOGIA}

El sistema se simulo aplicando el elemento finito en condiciones no lineales del material con una ley de endurecimiento isotrópico, por la evidencia física de la deformación plástica en el entorno de las laminaciones, la cual es asociada a las altas presiones que produce el Hidrógeno molecular en el interior del defecto, además de la presión de operación del tubo. Para elaborar los modelos, se considero un tubo de $610 \mathrm{~mm}$ de diámetro exterior con dos distintos espesores, uno de $15.8 \mathrm{~mm}$ y otro de $25.4 \mathrm{~mm}$. Se aplicaron las propiedades de un acero API 5L-X52 y se simulo el comportamiento en el régimen elastoplástico. Se consideraron las presiones en el interior de las laminaciones defecto, además de la presión interna del tubo. Los resultados se muestran en forma de mapas de distribución de los esfuerzos y se determina la presión que causa cedencia (PY) y la presión que hace alcanzar la resistencia máxima del material (PS). Estableciendo como criterio de falla la extensión de laminación a la que se alcanza la PS, se determinó que la extensión circunferencial de las laminaciones simples y las escalonadas que llevaría a la falla a un tubo con presión interna y se determinó el ángulo de deflexión de la grieta de interconexión de dos laminaciones no coplanares que se aproximan entre sí.

\section{Formulación de la no linealidad}

Una diferencia importante entre las relaciones correspondientes a los rangos elástico y plástico consiste en que dado un estado de esfuerzos puede calcularse la deformación mediante relaciones finitas como la ley de Hook, esto es para el primer rango, en cambio en el rango plástico las deformaciones no están en general determinadas unívocamente por los esfuerzos, sino que deben tratarse con relaciones diferenciales incrementales. La teoría de la plasticidad provee las relaciones matemáticas que caracterizan la respuesta de los materiales elasto-plasticos. Los componentes de la teoría de la plasticidad son: Criterio de fluencia, Regla de flujo, Regla de endurecimiento. Los infinitos estados de esfuerzo que determinan la entrada del material en el campo plástico, constituyen una superficie cerrada denominada superficie de fluencia. La expresión de la superficie de fluencia es en general una función de las variables de estado dada por:

$f\left(\sigma_{i j}, \varepsilon_{i j}^{p}, k\right)=0$

Donde $k$ es un coeficiente que mide el endurecimiento por deformación. Se emplea el criterio de fluencia de von Mises, lo que determina cuando se inicializa la cedencia.

$\sigma_{e}=\sqrt{\frac{1}{2}}\left[\left(\begin{array}{ll}\sigma_{1} & \sigma_{2}\end{array}\right)^{2}+\left(\begin{array}{ll}\sigma_{2} & \sigma_{3}\end{array}\right)^{2}+\left(\begin{array}{ll}\sigma_{3} & \sigma_{1}\end{array}\right)^{2}\right]^{1 / 2}=f(\{\sigma\})$

La deformación total dada por la ecuación 3, esta compuesta por los incrementos de la deformación elástica $\varepsilon^{\mathrm{el}} \mathrm{e}$ incrementos de la deformación plástica $\varepsilon^{\mathrm{p}}$ :

$\varepsilon=\varepsilon^{e l}+\varepsilon^{p}$

En el régimen elastoplástico las relaciones esfuerzo deformación están dadas por:

$d \sigma=E^{\tan } d \varepsilon$

Donde $E^{\text {tan }}$ es la pendiente de la curva esfuerzo deformación. Se considera que la superficie de carga se expande conservando su forma, es decir, es la superficie de fluencia correspondiente a un material con solo endurecimiento isotrópico Zienkiewick (1995). El historial de la deformación plástica de un metal es a menudo caracterizado por el trabajo plástico dado por $\mathrm{w}_{\mathrm{p}}$ :

$w_{p}=\int\{\sigma\}^{T}\left\{d \varepsilon^{p}\right\}$ 
Para este caso de estudio se considera la plasticidad asociativa, ya que el flujo plástico es normal a la superficie de cedencia. El problema general se formula en función del parámetro D (desplazamiento), en cada incremento el esquema iterativo anterior se ejecuta hasta que se alcanza la convergencia ó el máximo número de iteraciones. En cada incremento la matriz de rigidez tangente $\mathrm{K}_{\mathrm{T}}$ puede ser adaptada en cada iteración (Newton-Raphson) Ec. (6).

$\left.\left[K_{T}\right]\{\Delta D\}=\left\{F_{e}\right\} F_{n r}\right\}$

\section{Condiciones de la modelación}

Por la singularidad del esfuerzo en punta de grieta el modelo se construyó con elementos con 20 nodos y 3 grados de libertad por nodo. Se refinó la malla en los extremos de las grietas mediante elementos bi-cuadráticos para no afectar la sensibilidad del estudio. Se empleo el código comercial ANSYS V11.0. Los modelos geométricos y las variables de modelación se muestran en la figura 3.

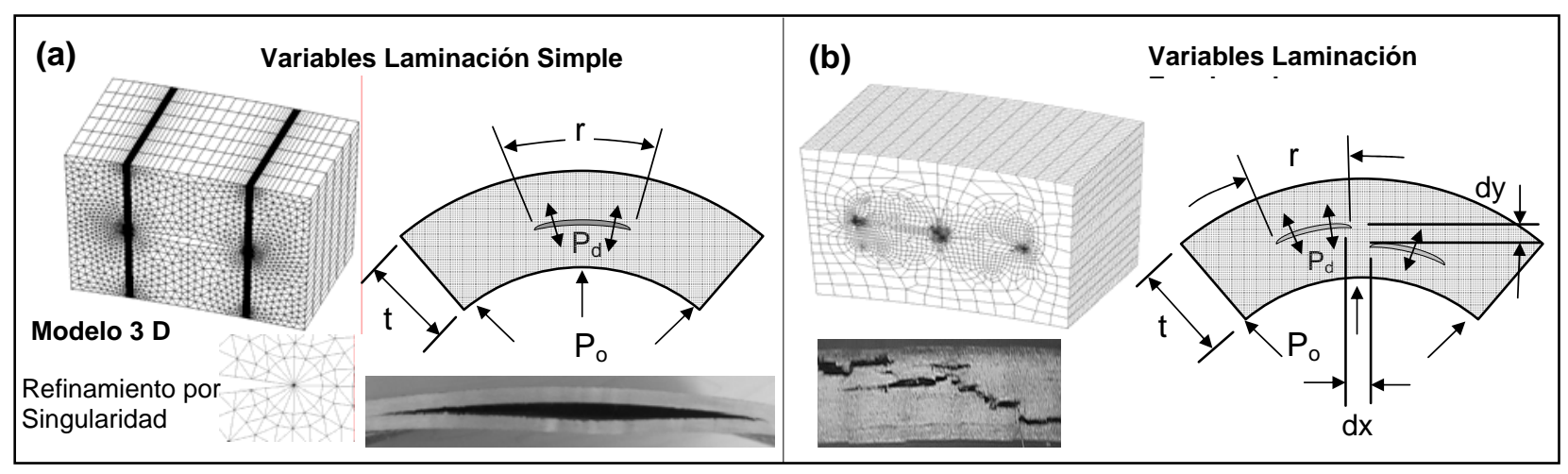

Fig. 3: Modelo mediante elementos sólidos, Laminación Simple(a), Laminación escalonada (b).

Las condiciones de frontera permiten la libre expansión del tubo mediante la rotación al sistema coordenado cilíndrico con restricción impuesta a los nodos. Se aplico la curva esfuerzo deformación del acero API $5 \mathrm{~L}$ X52, que posee un esfuerzo de cedencia $\sigma \mathrm{ys}=358 \mathrm{MPa}$, un esfuerzo máximo $\sigma \mathrm{u}=$ $453 \mathrm{MPa}$, modulo de Young $\mathrm{E}=203 \mathrm{GPa}$ y relación de Poisson de 0.3. Se aplicaron pasos de carga para la presión en el tubo, de 0.1 hasta $1.7 \mathrm{MPa}$ y la presión interna de las laminaciones. La matriz de simulación se muestra en la tabla 1.

Tabla 1: Descripción geométrica de los casos de estudio para laminaciones simétricas

\begin{tabular}{|c|c|c|c|c|c|c|c|c|c|}
\hline \multicolumn{5}{|c|}{ Espesor $\mathrm{t}=15.875 \mathrm{~mm}$} & \multicolumn{5}{|c|}{ Espesor $\mathrm{t}=25.4 \mathrm{~mm}$} \\
\hline \multicolumn{2}{|l|}{$\begin{array}{l}\text { Laminación } \\
\text { Simple }\end{array}$} & \multicolumn{3}{|c|}{ Laminación escalonada } & \multicolumn{2}{|c|}{$\begin{array}{l}\text { Laminación } \\
\text { Simple }\end{array}$} & \multicolumn{3}{|c|}{ Laminación escalonada } \\
\hline No de caso & $\mathrm{r} / \mathrm{mm}$ & $\mathrm{NC}$ & $\mathrm{r} / \mathrm{mm}$ & $(d x) \&(d y)$ & $\mathrm{NC}$ & $\mathrm{r} / \mathrm{mm}$ & $\mathrm{N} \mathrm{C}$ & $\mathrm{r} / \mathrm{mm}$ & $(d x) \&(d y)$ \\
\hline 1 & 12.7 & 6 & 12.7 & & 11 & 12.7 & 16 & 12.7 & \\
\hline 2 & 25.4 & 7 & 25.4 & & 12 & 25.4 & 17 & 25.4 & \\
\hline 3 & 76.2 & 8 & 76.2 & $0.8 \mathrm{~mm}$ & 13 & 76.2 & 18 & 76.2 & $0.8 \mathrm{~mm}$ \\
\hline 4 & 101.6 & 9 & 101.6 & & 14 & 101.6 & 19 & 101.6 & \\
\hline 5 & 127 & 10 & 127 & & 15 & 127 & 20 & 127 & \\
\hline
\end{tabular}

\section{RESULTADOS}

Se presentan los resultados de los casos de estudio 1 y 6 , en el entendido que los resultados y conclusiones de los demás casos son similares y siguen las mismas tendencias. La distribución de los esfuerzos de von Mises se muestra en la figura 4. La figura 4(a) corresponde al punto justo antes de la cedencia, el cual se alcanza con una presión en el tubo de 0.7 MPa y una presión interna en las laminaciones de $46.4 \mathrm{MPa}$. El comportamiento mas allá de la cedencia (no lineal) y hasta que se 
alcanza la resistencia máxima se observa en la figura 4(b) y corresponde a una presión interna del tubo de 1.7 MPa y presión en la laminación de $265 \mathrm{MPa}$. Se observa que al aumentar la presión en la laminación, los esfuerzos máximos en la zona de la punta de grieta se extienden en dirección perpendicular, lo cual es un indicativo de una probable deflexión de la grieta. La distribución del esfuerzo en los extremos de la grieta corresponde a la distribución de esfuerzos en la zona plástica para Modo I reportada por Ikeda (1997).

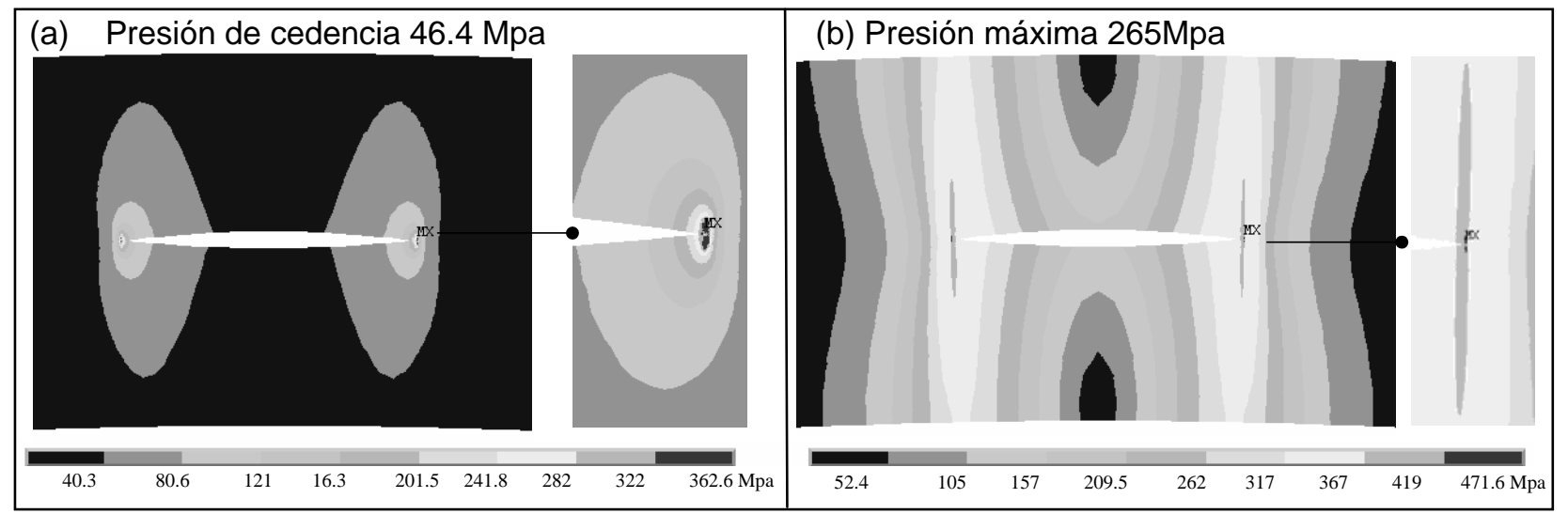

Fig. 4: Mapa del esfuerzo de v Mises en Mpa, correspondiente a una laminación simple caso 1.

La figura 5 muestra la dirección de los esfuerzos principales representados por vectores, aceptando que la orientación de la fractura en Modo I es perpendicular al esfuerzo principal máximo, lo cual se representa en las figuras por medio de los vectores que tienden a la horizontal por medio de líneas gruesas, es posible trazar la trayectoria de la deflexión de una laminación presurizada hasta alcanzar la resistencia máxima en el material circundante. En este caso, la línea roja muestra el resultado de este ejercicio; nótese la similitud con un caso real mostrado en la misma figura.

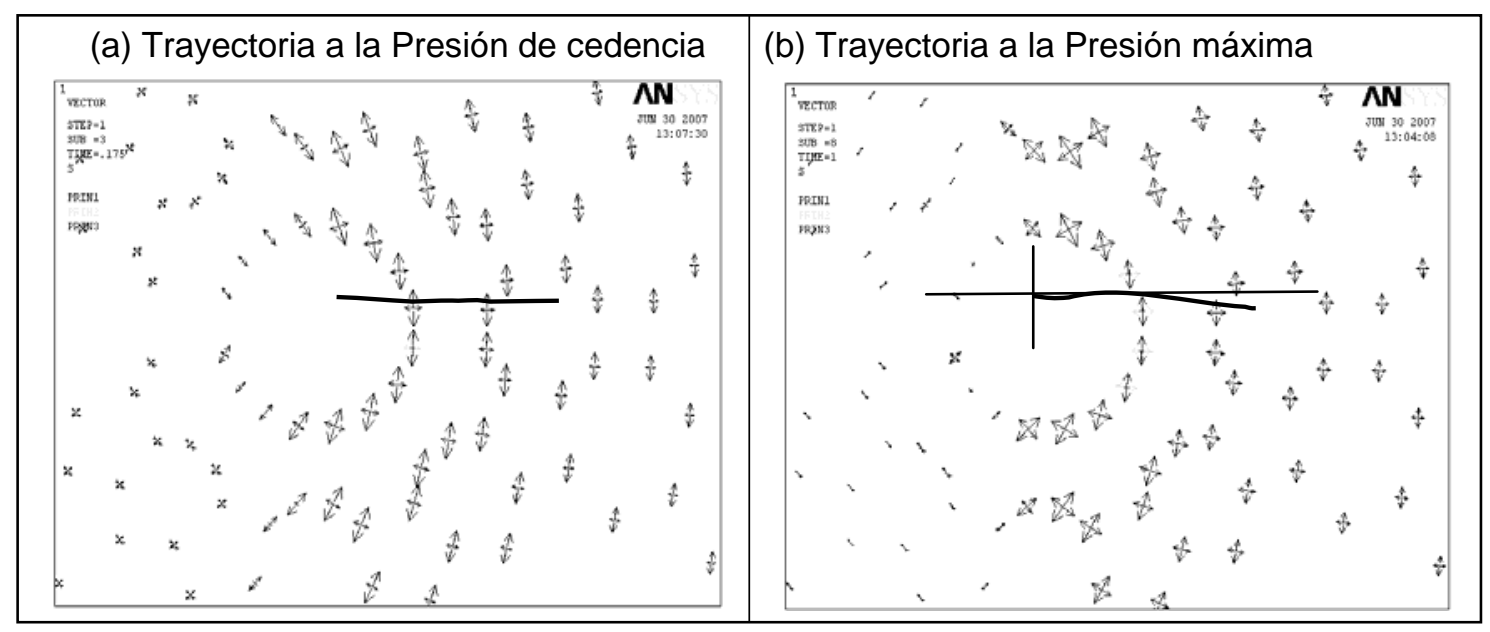

Fig. 5: Posible trayectoria de una laminación simple.

En los casos 1 al 5 y 11 al 15, se observa que mientras mayor es la extensión circunferencial de la laminación, el ángulo de deflexión crece. El gradiente del ángulo es de 1.5 grados por cada incremento de extensión circunferencial, lo que significa que para el caso 5, el ángulo de deflexión sea de 16 grados.

\section{Laminación Escalonada}

Bajo las mismas consideraciones de las laminaciones simples, para ilustrar los resultados de las laminaciones escalonadas, se presenta el análisis del caso 6 . En este caso, el esfuerzo máximo se presenta en las puntas de grieta de la región interlaminar, debido a la interacción de los campos de 
esfuerzos correspondientes a esta región, lo cual concuerda con el argumento del desarrollo del agrietamiento por la interconexión de grietas perpendiculares al eje de carga y la subsecuente fractura del ligamento. La figura 6(a) muestra los esfuerzos justo antes de la cedencia, la cual se alcanza con una presión en el tubo de 0.7 MPa y una presión en las laminaciones de $27.5 \mathrm{MPa}$. El campo de esfuerzos cuando se alcanza la resistencia máxima del material se observa en la figura 6(b), para una presión del tubo de 1.7 MPa y de 118 MPa en las laminaciones.

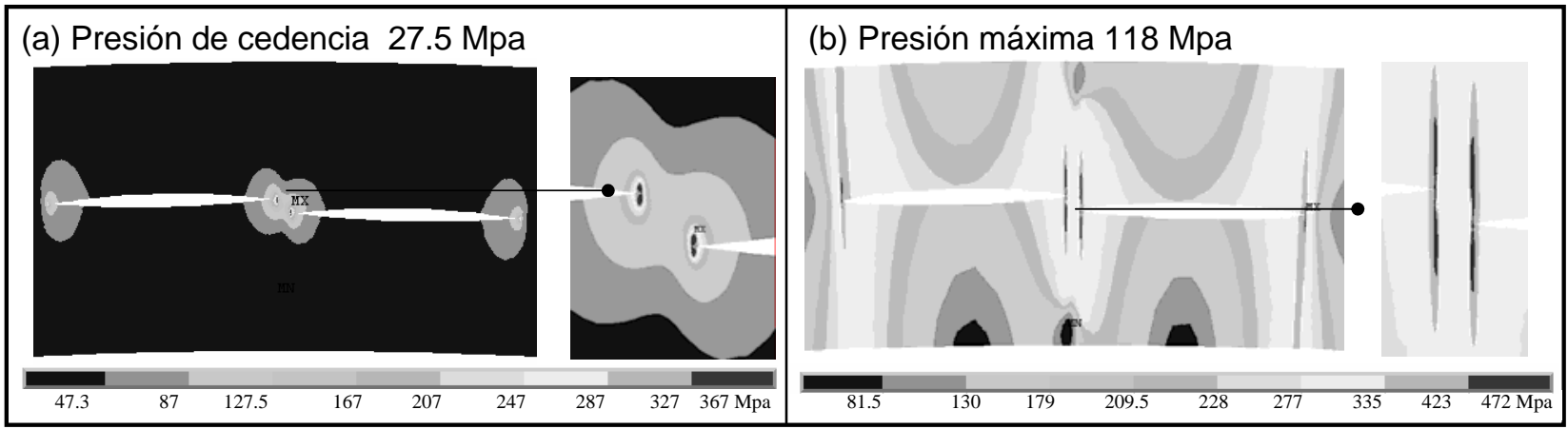

Fig. 6: Mapa del esfuerzo de Von Mises correspondiente a una laminación escalonada, Caso 6.

Para este caso de estudio, la posible trayectoria de interconexión de la grieta ocurre en la región interlaminar y se registra con un ángulo de 21 grados, como se muestra en la figura 7. Al incrementar la extensión circunferencial de las laminaciones se obtiene un gradiente del ángulo de deflexión de $2.5^{\circ}$, es decir que para el caso 10 el ángulo de deflexión es de 30 grados aproximadamente.

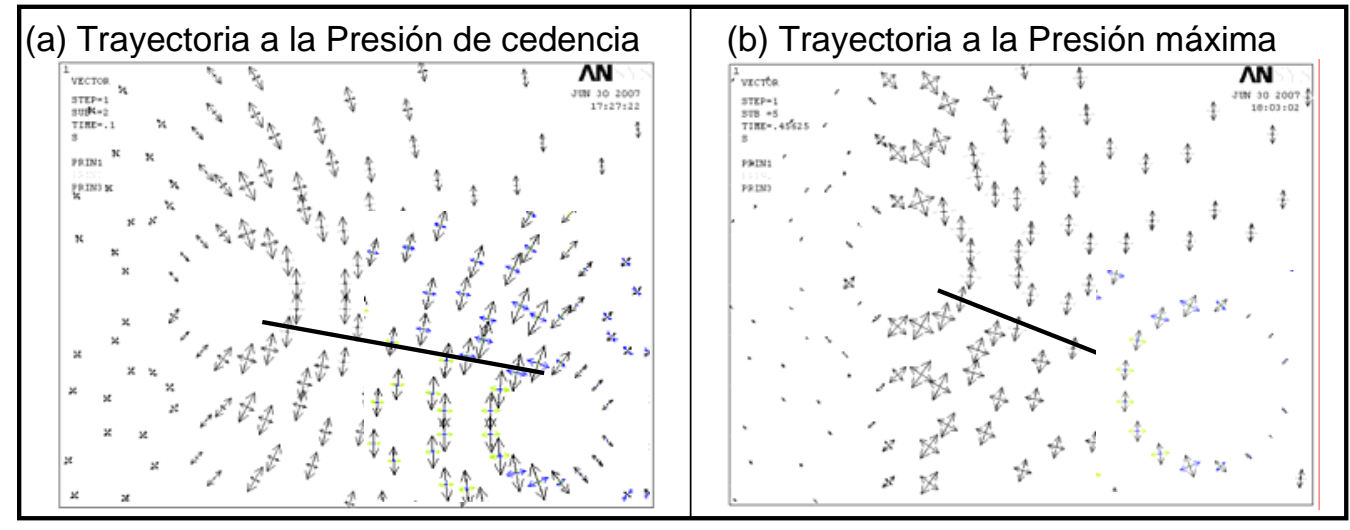

Fig. 7: Posible trayectoria de interconexión de una laminación escalonada

\section{DISCUSIÓN}

Uno de los objetivos de esta investigación fue calcular los valores de la presión interna en laminaciones, que produce cedencia y que hace alcanzar la resistencia máxima de material en la zona de la punta de grieta. A la presión que produce cedencia se le denominó PY y a la presión que hace alcanzar la resistencia máxima del material -acero API $5 \mathrm{~L}$ X52- se le denominó PS, esto con el fin de establecer las condiciones de deflexión de la punta de grieta y de interconexión de grietas escalonadas. En las figuras 8 y 9, se muestran los valores de PY y PS respectivamente, calculados por MEF en función de la extensión circunferencial de la laminación (recuérdese que en el caso de las laminaciones escalonadas la longitud de ambas es la misma), se observa que las PS son alrededor de 3 a 5 veces mayores que las PY, lo que indica que la fractura del ligamento, en las laminaciones escalonadas o la deflexión de laminaciones simples requiere de presiones mucho mayores que las necesarias para expandir la grieta, que las que corresponden a las PY.

En las figuras 8 y 9 se observa que la tendencia general es que PY y PS disminuyen al incrementarse el tamaño de las laminaciones; esta tendencia sigue una función potencia, donde el exponente es menor de uno, esto hace que para tamaños de laminación mayores a $76.2 \mathrm{~mm}$, la 
curva tenga una reducción significativa en la pendiente; de ahí que sea razonable esperar que laminaciones de más de $76.2 \mathrm{~mm}$ evolucionen más fácilmente, ya sea por deflexión o por interconexión con otras laminaciones, pues un pequeño incremento de presión en las grietas produce expansión o extensión del agrietamiento.

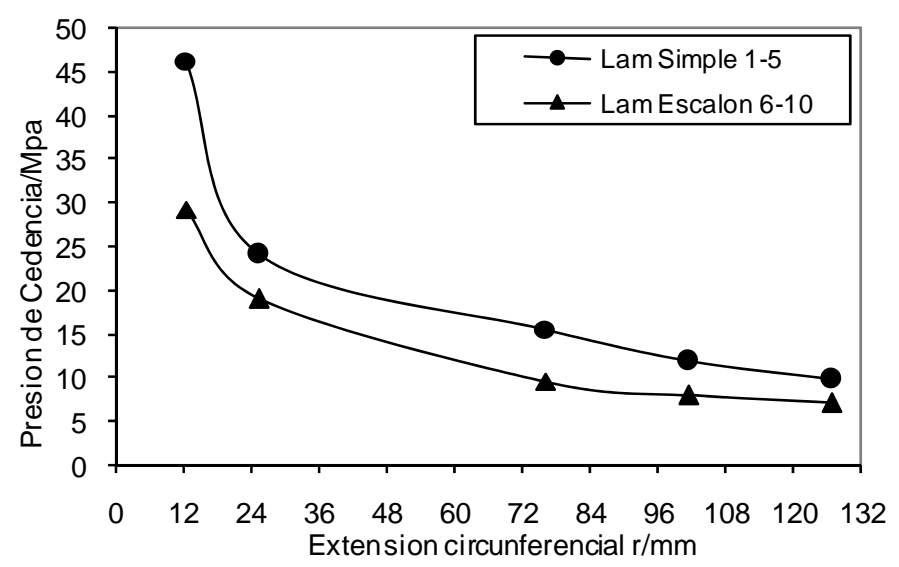

Fig. 8: Variación de la presión de cedencia, en función de la extensión circunferencial.

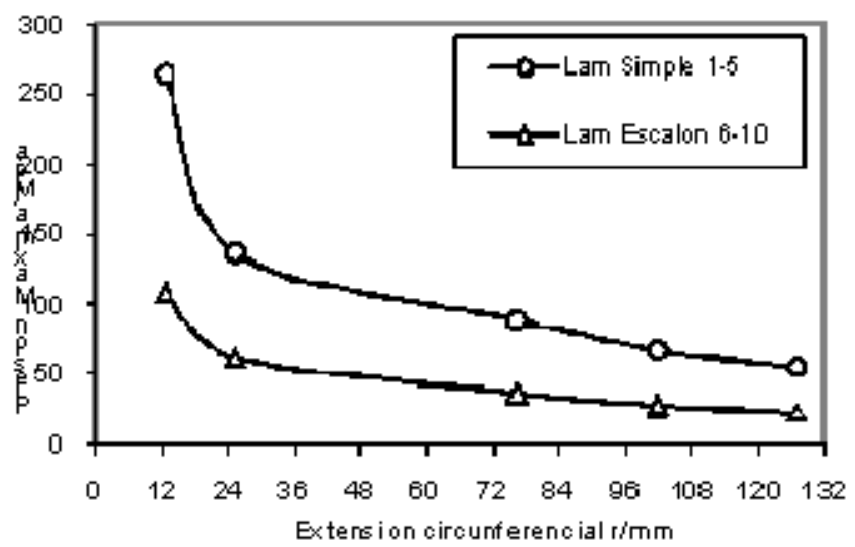

Fig. 9: Variación de PS en función de la extensión circunferencial de las laminaciones.

Otro aspecto de interés es el efecto del espesor en el comportamiento mecánico de laminaciones escalonadas; en este trabajo se tuvo la oportunidad de revisar éste efecto en combinación con la longitud de laminación. La figura 10 muestra que la PS aumenta ligeramente al aumentar el espesor, tal como se ha reportado en estudios previos de González (2004).

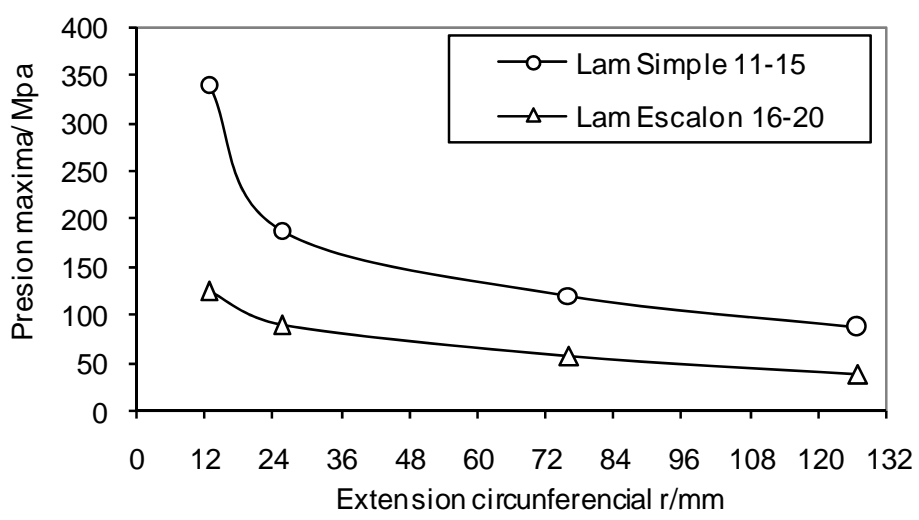

Fig.10: Función de la presión versus la extensión de la laminación en un tubo de 25.4mm de espesor.

La figura 11 muestra la variación del ángulo de deflexión de la grieta al incrementarse la extensión circunferencial. Se observa que para las laminaciones escalonadas el ángulo es mayor que en las 
laminaciones simples, debido a la interacción de los campos de esfuerzos en la región interlaminar. Las tendencias son casi paralelas en ambos casos, para extensiones de laminación de 25 a $80 \mathrm{~mm}$, la diferencia en el valor de $13^{\circ}$ en laminaciones simples y $23^{\circ}$ para laminaciones escalonadas.

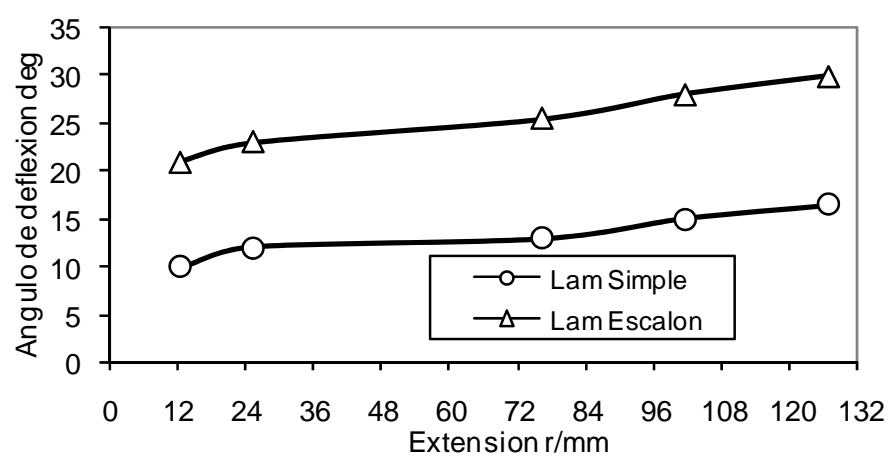

Fig.11: Angulo de deflexión en laminación simple versus extensión, tubo de 15.8 mm de espesor

\section{CONCLUSIONES}

El análisis aplicado permitió establecer los valores de las presiones límite al valor frontera de las propiedades del material API5LX52 (cedencia y ultima), en ductos que presentan laminaciones tanto simples como escalonadas, determinando que las laminaciones simples cuya extensión circunferencial va de 24 a $127 \mathrm{~mm}$ soportan 50\% más de presión de cedencia que las laminaciones escalonadas, y el 100\% más de presión ultima.

La extensión de laminaciones escalonadas requiere menor presión, por lo tanto las hace de mayor riesgo una vez en servicio. Esta condición se hace mas evidente para laminaciones de mas de 76.2 $\mathrm{mm}$ de longitud, por lo que se concluye que una laminación en servicio de tamaño mayor a $76.2 \mathrm{~mm}$ puede considerarse severa. También se predijo el ángulo de deflexión de la extensión de una laminación simple y el ángulo de la grieta de interconexión de dos laminaciones no coplanares que se aproximan entre sí, encontrando que el ángulo es mayor en grietas no coplanares. Estos resultados tienen buena concordancia con los ángulos observados en la realidad.

El espesor del ducto juega un papel importante ante la presencia de laminaciones simples, ya que para el caso de un espesor de $15.8 \mathrm{~mm}$ la presión ultima es $30 \%$ menor a la que soporta esa misma laminación simple en un ducto de espesor de $25.4 \mathrm{~mm}$. Esta sensibilidad al espesor es menor para el caso de las laminaciones escalonadas ya que la diferencia de espesor enmarca solo un $10 \%$ en valor de la presión al incrementar el espesor.

\section{REFERENCIAS}

Gonzalez J.L.; Analysis of non coplanar pressurized laminations in X52 steel pipes by non-linear finite element, The Journal of Pipeline Integrity: 3(3), 147-156 (2004).

Ikeda A.; On the Hydrogen Induced Cracking of Pipeline Steel Under Wet Hydrogen Sulfide Environment, second Intl. Cong. Hydrogen in Metals, 4-7, Paris June 6-11 (1997).

Itou S.; 3D Dynamic Stress Intensity Factors Around Two Parallel Square Cracks in Infinite Elastic Mediun Under Impact Load, Archive of Applied Mechanics: 71, 54-71 (2001).

Kratzig W.B.; Assessment of structural damage and failure, Archive of Applied Mechanics: 71, 1-15 (2001).

Makkio I.; The Extension of Hydrogen Blister-Crack Array in pipeline Steels, Metallurgical Transactions: 9A, 1581-1590 (1978). 
Morales A.; Analysis of Laminations in X52 Steel Pipes by Nonlinear by Finite Element, Journal of Pressure Vessel Technology: 130, 21-30 (2008).

Moussa W.A.; The Interaction of Two Parallel Semielliptical Surface Cracks Under Tension and Bending, Journal of Pressure Vessel Technology: 121, 323-326 (1999).

Noda N.A.; Variation of the stress intensity factor along the crack front of interacting semi-elliptical surface cracks; Archive of Applied Mechanics: 71, 43-52 (2001).

Zacaria M. y Davies T.; Formation and Analysis of Stack Cracks in Pipeline Steel, Journal of Materials Science: 28, 322-328 (1993).

Zienkiewick O.C.; Finite Element method, 24-54, Mc Graw Hill, Spain (1995). 
